\title{
Los concheros de Baja California y sus perspectivas de investigación
}

\author{
Agustín Téllez Duarte \\ Facultad de Ciencias Marinas \\ Universidad Autónoma de Baja California
}

\begin{abstract}
Resumen
El presente artículo pretende enfatizar sobre la presencia de conchas en sitios arqueológicos, los cuales nos proporcionan una gran cantidad de información cultural sobre los asentamientos humanos, particularmente en los concheros de Baja California. En Baja California se localizan en forma intermitente a todo lo largo de sus costas, tanto en el golfo de California como en el Pacífico. Con este proyecto se pretende rescatar aquellas colecciones que aún puedan conservarse, considerando que las excavaciones de Palmer fueron muy productivas a pesar de haber sido relativamente pequeñas. Se espera que este primer intento de excavación arqueológica-científica de un conchero proporcione datos relevantes para comprender más ampliamente la organización de las antiguas sociedades humanas de Baja California, antes de que se pierdan totalmente los testimonios legados. Y a su vez, motive un mayor interés y apoyo en el estudio de los casi ignorados concheros.
\end{abstract}

Palabras clave: concheros, asentamientos humanos antiguos, Baja California, excavaciones arqueológicas, delta del Río Colorado.

\begin{abstract}
This article researches on the presence of shells found in archeological sites, which have offered a huge amount of cultural information about human settlements, in particular those where there are shell deposits (concheros) at Baja California. Shell deposits are intermittently located along the coasts of Baja California, on the Gulf of California, as well as in the Pacific Ocean shores. This project pretends to rescue those collections that could still be kept, taking into account that Palmer excavations were pretty productive; even though they were relatively small. It is expected to obtain outstanding information from this scientificarcheological excavation performed in shell deposits. The idea is that this information will help to better understand the kind of organization that antique human societies of Baja California had before the inherited and existing testimonies completely disappear and at mean time to foster more interest and support to continue researching on the almost ignored shell deposits.
\end{abstract}

Keywords: Shell deposits, antique human settlements, Baja California, archeological excavations, Colorado River delta. 


\title{
LOS CONCHEROS DE BAJA CALIFORNIA Y SUS PERSPECTIVAS DE INVESTIGACION
}

\author{
Por \\ Agustín Téllez Duarte*
}

\section{INTRODUCCION}

Las conchas de los moluscos han desempeñado un papel muy variado a lo largo de la historia. Originalmente, han sido utilizadas comofuente alimenticia, dejando como testimonio de esta actividad extensos depósitos de conchas en las zonas costeras. Otros usos importantes fueron como objeto de trueque, ornamental, ritual y utilitario en diversas actividades de la vida cotidiana. Por estas razones, la presencia de conchas en sitios arqueológicos nos proporciona una gran cantidad de información cultural sobre los antiguos asentamientos humanos, acerca de lo cual se pretende enfatizar en el presente artículo, particularizando en los concheros de Baja California.

Aunque no existe una definición formalizada de la palabra "conchero" ésta conlleva la existencia de grandes depósitos de conchas de moluscos de agua dulce o marina, producto de la actividad humana. La palabra deriva de los mesolíticos del mar Báltico (Meinghan, 1969) y ha sido extensivamente aplicada tanto en Europa como en América, por la gran abundancia de estos sitios arqueológicos en casi todas las zonas costeras del mundo.

En Baja California se localizan en forma intermitente a todo lo largo de sus costas, tanto en el golfo de California como en el Pacífico, siendo más escasos y menos espectaculares los situados hacia las zonas montañosas, donde comúnmente se localizan en los pisos de resguardos o cuevas poco profundas. Debido a que no poseen la espectacularidad de las pinturas rupestres, que han caracterizado la arqueología bajacaliforniana, muy poco ha sidoel interés en estudiarlos, tanto en la península, como en todo el litoral mexicano (Mora, 1980). Por otro lado, el continuo avance de núcleos poblacionales y campos pesqueros, está causando su paulatina destrucción, aunada a la colecta de artefactos por coleccionistas y turistas.

\section{LA SELECTIVIDAD DEL RECURSO}

La información básica que puede ser obtenida de los depósitos de conchas está referida a la selectividad.del recurso como fuente alimenticia, comparando porcentualmente las distintas especies presentes en un conchero. Por lo general, vamos a encontrar que existe preferencia en la colecta de una sola especie, la cual puede ser abundante en el área. Este es el caso del mejillón Mytilus

* Paleontólogo investigador de tiempo completo de la Facultad de Ciencias Marinas de la UABC. 
californianus, el cualllega a conformar hasta el $90 \%$ de las especies presentesen los concheros de la península. Esto es consecuencia de que el mejillón formara densos mantos en la zona intermareal rocosa, constituyendo el grueso de la biomasa de moluscos de esta zona donde son accesibles y fácilmente colectados. También importantes en orden decreciente son los abulones (Haliotis spp.), dentro de los cuales el más común en los concheros es el abulón negro $H$. cracherodii, por ser el más abundante y accesible de su especie en la zona intermareal. En menor porcentaje se colectaban las lapas Lottia gigantea, Fissurella volcano, Collisella spp. y los caracoles Astraea spp. y Tegula spp.

A manera de metodología, al comparar porcentualmente los bivalvos o almejas con respecto a los gasterópodos o caracoles, es necesario considerar las valvas más abundantes, ya sea derechas o izquierdas, que aún conservan el umbo, para asegurar que éstas pertenezcan a un solo especimen. Es de esperar que si los bivalvos son consumidos en el mismo sitio donde se colectan, en los montículos de desecho deberán existir aproximadamente el mismo número de valvas derechas e izquierdas. Esto último debe ser probado estadísticamente para dar más soporte a las interpretaciones.

En ocasiones es posible encontrar, asociados a los concheros huesosde mamíferos marinos y continentales, aves y peces, lo que sugiere que constituian otra importante fuente alimenticia, considerando que cada gramo de hueso puede representar hasta 100 gramos de conchas de moluscos (Cook y Treganza, 1980).

Para la extracción del recurso se requeríael uso de cierta técnica que va desde la simple colecta superficial, hasta el uso de artefactos, comúnmente de piedra. Es por ello que en la mayoría de los concheros se encuentran navajas y raspadores líticos que eran utilizados para desprender y desconchar los moluscos, preservándose en muchas de las conchas las huellas de la extracción, ya sea como rayas en el interior de las conchas, o como muestras próximas a la apertura sifonal de los bivalvos.

\section{LOS MOLUSCOS Y EL COMERCIO}

Las atractivas formas y colores de las conchas de los moluscos han dado por resultado que comúnmente fueran utilizadas como objeto artesanal para la producción de artesanías ornamentales. Otra característica importante que presentan es la facilidad para ser trabajadas, ya que en muchas ocasiones se requieren unos cuantos cortes y modificaciones de la forma original para darle la figura deseada; en muchos casos, sólo una simple perforación es suficiente para ser utilizada como pendiente. Además, su forma natural permite su uso doméstico, como cucharas, raspadores o simple recipiente, no requiriendo de modificación alguna. Todas estas características han hecho que las conchas hayan sido un objeto de trueque frecuente. Quizás la concha del Pacífico más preciada para los fines antes mencionados haya sido la del abulón, la misma se ha encontrado en sitios arqueológicos de Arizona y Chihuahua. Es por ello que la costa del Pacífico debió haber sido un importante punto de exportación de estas conchas. Sin embargo, quizás lo más sorprendente sea la presencia de 
conchas de Estrombussp. del golfo de México en sitios arqueológicos del golfo de California. Incluso en el Museo Regional de Mexicali se conserva una trompeta de Strombus con boquilla de asfalto; sin embargo, las rutas de intercambio de estos objetos requieren de estudios más detallados. No se ha reportado la presencia de conchas del golfo de California en los concheros del Pacífico, sólo hasta las zonas montañosas, donde se han encontrado Turritella y Glicymeris, aunque no en cantidades apreciables, pero significan un traslado de hasta $160 \mathrm{~km}$.

\section{SIGNIFICADO AMBIENTAL}

La distribución geográfica de los moluscos está determinada por límites naturales impuestos por varios factores físico-químicos, siendo el más importante la temperatura. Las grandes áreas de distribución impuestas por estos factores se denominan provincias. El provincialismo de los moluscos permite reconocer, de manera aproximada, el área fuente de donde fueron extraídos para el establecimiento de rutas de intercambio comercial. Así, para el Pacífico tenemos especies características desde punta Concepción, Estados Unidos, hasta bahía Magdalena, Baja California Sur, quedando englobadasdentro de la provincia californiana. Especies típicas de esta provincia sonel abulón Haliotis spp. y la almeja pismo Tivela stultorum.

A niveles locales la distribución vertical y horizontal de los moluscos está regida por las características del sustrato, temperatura, salinidad, oxígeno disuelto y nutrientes principalmente. Es por ello que existen hábitats definidos donde pueden funcionar eficientemente, de tal forma que las especies presentes en un conchero pueden indicar el tipo de esfuerzo requerido para su extracción, ya que si fueron colectados en la zona intermareal, se requirió del buceo a distintas profundidades.

Información adicional con respecto al ambiente nos la proporcionan las líneas de crecimiento de las conchas, ya que ellas registran la estacionalidad de la recolección. Esto es válido sólo parazonas templadas o frías, donde el espesor de las líneas de crecimiento varía de acuerdo con la estación del año. En el caso de las zonas tropicales, no es posible utilizar las líneas de crecimiento debido a que éste es continuo durante todo el año, por lo cual es difícil definir cambios estacionales en la precipitación de carbono de calcio.

La estacionalidad en la colecta puede determinarse mediante la obtención de las paleotemperaturas registradas en las líneas terminales de las conchas, ya que ésta nos indica la temperatura ambienta! al momento de la muerte del organismo, y se determina mediante la razón de isótopos de 016/018 en el carbonato de la concha. Si para varias muestras se encuentra que la temperatura es la misma, esto nos indicará una estacionalidad en la colecta; en caso contrario, significará colectas durante todo el año.

Una ventaja adicional en el estudio de los concheros es la de poder determinar la edad mediante fechado radiométrico, lo que en un estudio estratigráfico nos 
llevará a establecer los cambios culturales con respecto al tiempo, muchos de los cuales pueden estar relacionados con cambios ambientales. La metodología y limitaciones del fechado radiométrico son dadas por Shackleton (1980).

\section{EL CONCHERO DE LA FACULTAD DE CIENCIAS MARINAS}

La Facultad de Ciencias Marinas se encuentra situada sobre uno de los abundantes concheros de la costa del Pacífico de Baja California. Durante las obras de construcción de edificios, las continuas remociones del suelo han permitido colectar algunos artefactos y hacer algunas observaciones superficiales del significado arqueológico de los depósitos. El sitio nunca ha sido excavado con un estudio formal.

Como todos los concheros distribuidos a lo largo de la provincia californiana, la especie más abundante es el mejillón Mytilus californianus, al que se encuentran asociados en menos cantidad las lapas Lottia gigantea, Fisurella volcano, Collisella spp. y el abulón Haliotis cracherodii. Un poco menos comunes son los gasterópodos Astraea spp., Tegula spp. y las placas del quitón Lepidozona sp. Todos estos moluscos indican que la colecta se llevaba a cabo en la zona rocosa intermareal que domina la costa de la bahía de Todos Santos. Nunca se han encontrado objetos de concha manufacturada; aunque existe esta posibilidad, la mayor parte de la colecta era principalmente con fines alimenticios.

La escasez de huesos de peces parece indicar que la pesca era una actividad poco frecuente, que posiblemente se restringía a la captura de los que quedaban atrapados en las pozas de mareas. Más importante debió șer la caza de pequeños mamíferos o aves, lo que se infiere de las pequeñas puntas de flecha que se han encontrado en los depósitos, y ocasionalmente cazaban mamíferos marinos, actividad poco frecuente por lo escaso de los huesos de éstos. El aprovechamiento de pieles de mamíferos como vestido puede inferirse por la presencia de buriles líticos.

La presencia de manos de mortero sugiere que otra actividad complementaria en la dieta alimenticia era la colecta de plantas y frutos silvestres. Sin embargo, los artefactos más comunes son los raspadores, utilizados en la extracción de la carne de los moluscos cuyas huellas es frecuente observar en los bordes e interior de las conchas.

Los tipos de artefactos líticos y la presencia de alfarería sitúan a este grupo como perteneciente al yumano III o diegueño (Kinder, 1970), o de acuerdo con la nomenclatura de Schoroeder (1979) la hakataya. Evidencia adicional la representan los materiales de importación para la manufactura de artefactos, como el ópalo delárea de La Misión, el cuarzo de las rocas batolíticas peninsulares y la obsidiana, que probablemente la importaban del área de Jacumba, E.U.A., pero en general, fueron elaborados con materiales pétreos de la localidad, como basaltos andesíticos. 
Un aspecto importante del sitio es el hallazgo, por Katzuo Nishikawa, de una quijada humana hacia la base del conchero, de la cual no se ha determinado su edad. De ser realmente una quijada antigua, sería un hallazgo sumamente importante, puesto que el grupo diegueño se caracterizó por practicar la cremación, lo que hace sumamente difícil encontrar restos óseos de éstos.

El conchero de la Facultad de Ciencias Marinas se encuentra en la actualidad alterado por las continuas excavaciones, sin embargo, a lo largo de la península son sumamente abundantes, y es necesario estudiarlos antes de que sean destruidos por el constante crecimiento de los centros poblacionales.

Actualmente se encuentra en proceso un proyecto del Instituto de Investigaciones Sociales de la.UABC para la excavación en un conchero de bahía de los Angeles. Este proyecto en sí representa más bien un rescate cultural de lo poco que aún puede recuperarse de un sitio excavado a finales del siglo pasado por Palmer. Producto de esta excavación se obtuvo una colección poco común de objetos, artefactos e incluso restos óseos que actualmente se conservan en el Museo Smithsoniano de Estados Unidos y que fueron dados a conocer en un trabajo publicado por Massey y Osborne (1968). Como este sitio es uno de los escasos manantiales perennes en el árido paisaje bajacaliforniano, los habitantes de bahía de los Angeles se surten de aguas de él, por lo cual han hecho frecuentes excavaciones y remociones del terreno. Por ello, el sitio se encuentra alterado. Con este proyecto se pretende rescatar lo que aún puede conservarse, considerando que las excavaciones de Palmer fueron muy productivas a pesar de haber sido relativamente pequeñas.

Esperemos que este primer intento de excavación arqueológico-científica de un conchero proporcione datos relevantes para comprender más ampliamente la organización de las antiguas sociedades humanas de Baja California, antes de que se pierdan totalmente los testimonios que nos legaron. Y a su vez, motive un mayor interés y apoyo en el estudio de los casi ignorados concheros. 


\section{BIBLIOGRAFIA}

BROTHWELL, D. y E. Higgs (eds). 1980. Ciencia en arqueologia. Fondo de Cultura Económica. México.

IKE, D. 1978. Natural History of the Coronados Islands, Baja California, México. San Diego Association of Geologists.

KILLINGLEY, J.S., y Berger, W.G. "Stable Isotopes in a Mollusk Shell Detection of Upwelling Events". Science. 205.

KINDER, D.W. 1970. "Early Inhabitants of Southern California". En: Allison, E.C.; Acosta, M.G.; Fife, D.L.; Minch, J.A. y K. Nishikawa(eds). Pacific Slope Geology of Northern Baja California and Adjacent Alta California. Pacific Sections.

MASSEY, W. y C.M. Osborne. 1968. "A Burial Cave in Baja California: The Palmer Collection, Mexico". Pacific Coast Archaelogical Society. Quarterly. 6 (8).

MEIGHAN, C.W. 1980. "Los moluscos como restos de alimentos en sitios arqueológicos”. En: Brothwell y Higgs (eds). Ciencia en arqueología. Fondo de Cultura Económica.

MORA Echeverría, J.L. 1980. "Nuevo procedimiento de dotación arqueológica”. Boletín de antropología americana. № 2. Instituto Panamericano de Geografía e Historia.

SHACKLETON, N.J. 1980. "Los moluscos marinos en arqueología". En: Brothwell y Higgs (eds). Ciencia en arqueología. Fondo de Cultura Económica. 